\title{
The effects of dissonance and reward on perceptual distortion
}

\author{
DONALD R. YELEN \\ Washburn University of Topeka, Topeka, Kans. 66621
}

\begin{abstract}
The present experiment was concerned with the effect of dissonance and the presence or absence of reward on perceptual distortion. Dissonance was aroused by persuading students to make inconsistent psychophysical judgments. The inconsistent judgments were rewarded in one group and not rewarded in another group. Comparisons with a control group suggested that the students in both the rewarded and nonrewarded groups reduced dissonance by distorting their perception of the psychophysical stimuli during postdissonance trials.
\end{abstract}

Festinger (1957) has indicated that inconsistent or incompatible items will arouse dissonance in individuals and that the individuals will attempt to reduce this dissonance by changing their attitudes, by changing their behavior, or by changing their perception of the environment. Festinger \& Carlsmith (1959) have shown that the amount of dissonance aroused is likely to be affected by the amount of the reward given in the situation. Numerous studies (e.g., Rosenberg, 1965; Helmreich \& Collins, 1968) have investigated the effects of dissonance and amount of reward on changes in attitudes. The relationship between dissonance, amount of reward, and perceptual changes, however, has received little experimental attention.

The present experiment was designed to determine the effects of dissonance and the presence or absence of reward on perceptual distortion. Two groups of students were persuaded to make psychophysical judgments that would arouse dissonance. That is, the students were persuaded to make judgments that were inconsistent or incompatible with the stimuli presented. The students in one group were rewarded after their inconsistent judgments; the students in the second group were not rewarded. Students in the nonrewarded group could, according to Festinger (1957), reduce or eliminate the aroused dissonance by distorting their perception of the stimuli used in the psychophysical task. That is, by distorting the stimuli so that the stimuli would appear to be consonant with their inconsistent judgments, these students could reduce the dissonance aroused by their inconsistent judgments. It was expected, therefore, that psychophysical judgments made after the arousal of dissonance would reflect a distortion of the psychophysical stimuli. Students that were rewarded following their inconsistent judgments could, according to the arguments presented by Festinger \&
Carlsmith (1959), use the rewards to justify these judgments and, in this way, eliminate or reduce the dissonance aroused by the inconsistent judgments. Since dissonance could be reduced in this manner, there would be little or no reason for the rewarded students to distort their perception of the psychophysical stimuli. It was expected, therefore, that the psychophysical judgments made after the arousal of dissonance by the students in the rewarded group would show less distortion of the stimuli than the judgments made by students in the nonrewarded group.

\section{METHOD}

Experimental Conditions

Pairs of students in two experimental groups and one control group judged a vertical-horizontal illusion under predissonance, dissonance, and postdissonance conditions.

In the two experimental groups, one member of each pair of students was chosen to serve as a pseudoconfederate, and the other member was assigned to the role of an $O$. The pseudoconfederates were given deceptive instructions indicating that the experiment was concerned with the influence of one person's judgments on the judgments that another person would make. These students were then asked to cooperate by making false judgments during the second phase of the experiment. After the pseudoconfederates agreed to cooperate, they were instructed that they were free to indicate that either the vertical or horizontal line was longer during the pre- and postdissonance phases of the experiment, but they were asked to always say that the horizontal line was longer than the vertical line during the dissonance phase of the experiment. During the dissonance phase of the experiment, horizontal lines were presented which were noticeably shorter than the vertical line. Thus, the pseudoconfederates' judgments during the dissonance phase of the experiment were inconsistent or incompatible with the stimuli presented. In one experimental group, dissonance with reward (D-R), the pseudoconfederates' inconsistent judgments were followed by verbal reinforcement. In the second experimental group, dissonance with no reward (D-NR), the pseudoconfederates' inconsistent judgments were not reinforced.

The Os in the two experimental groups served two functions: First, they increased the credibility of the deceptive instructions given to the pseudoconfederates; and second, they provided an audience for the inconsistent judgments made by the pseudoconfederates.

The control group was used to determine the effect of practice on the vertical-horizontal illusion. In the control group the students chosen to be counterparts of the pseudoconfederates did not receive deceptive instructions and were not asked to make false judgments during the second phase of the experiment. The Os in the control group were treated exactly the same as the Os in the two experimental groups.

The dependent measure was the proportion of times that a horizontal line of varying length was judged to be longer than a vertical line during pre- and postdissonance trials.

\section{Subjects}

Thirty-nine pairs of introductory psychology students participated in the present experiment. Seven pairs of male students and six pairs of female students were assigned to each of the two experimental groups and to the control group.

\section{Apparatus}

A Lafayette Instrument Co. vertical-horizontal illusion board was used. The vertical line served as the standard stimulus and was $23 \mathrm{~cm}$ long. The horizontal line was used as the variable stimulus. During pre- and postdissonance trials, 11 different horizontal line lengths were used, ranging in length from 17.0 to $22.0 \mathrm{~cm}$ in $.5-\mathrm{cm}$ steps. During dissonance trials, three horizontal lines were used: $15.5,19.5$, and $23.5 \mathrm{~cm}$ in length. The illusion board was housed behind a sliding screen. In the closed position the screen prevented the students from observing the adjustments in the length of the horizontal line. When the screen was in the open position, the vertical and horizontal lines were exposed to the students.

A double-throw switch, with one position labeled vertical and the other position labeled horizontal, was mounted on each of two typing tables. The switches, used by the students to indicate their judgments, activated lights located behind the illusion board. The two tables were 


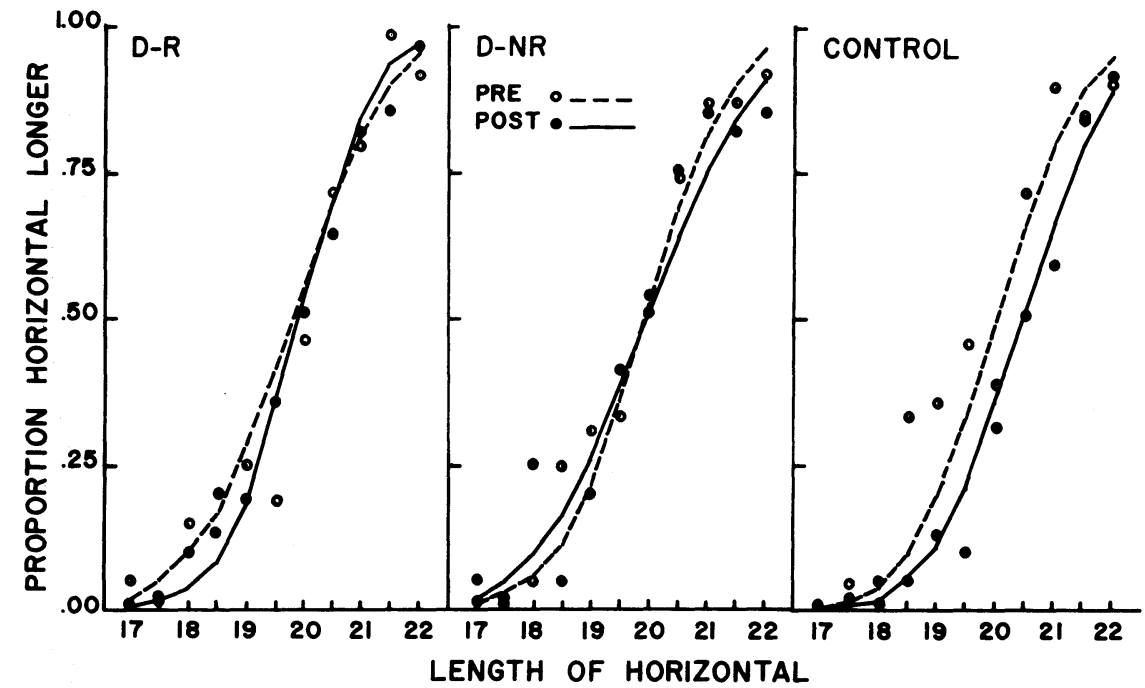

Fig. 1. The proportion of time each horizontal line was judged longer by pseudoconfederates in the three groups during pre- and postdissonance trials. The points show the observed proportions. The curves are best-fitting normal ogives.

placed three feet in front of the illusion board.

Procedure

A pair of students reported at the same time and were assigned to either one of the two experimental groups or to the control group. The Os in the two experimental groups were shown directly into the experimental room. The pseudoconfederates in the two experimental groups were taken to another room, given the deceptive instructions, and then returned to the experimental room. The counterpart of the pseudoconfederate and the $\mathrm{O}$ in the control group were both shown directly into the experimental room.

When both students were in the experimental room, they were seated at the tables facing the illusion board, given instructions for the psychophysical tasks, and then given a series of predissonance, dissonance, and postdissonance trials. During the pre- and postdissonance trials, E opened the screen, momentarily exposed the vertical and horizontal lines, and then closed the screen. When the screen was closed, both students were to indicate which line they judged to be the longest by turning the switch on their table to either the vertical or horizontal position. No equal judgments were allowed. The vertical line was presented with each of the 11 horizontal lines three times, in a random order, during both pre- and postdissonance trials.

During each dissonance trial, E exposed the vertical and horizontal lines and then called on one of the students to say which line was the longest. The students were instructed to respond by saying either "vertical" or "horizontal" aloud. The pseudoconfederates in both the D-R and D-NR groups were, of course, to follow the deceptive instructions and say horizontal each time they were called on to respond. A total of 30 dissonance trials were administered. The pseudoconfederates and their control counterparts were called on to respond during 15 of these trials. The vertical line was presented with a $15.5-\mathrm{cm}$ horizontal line nine times, with a $19.5-\mathrm{cm}$ line five times, and with a $23.5 \mathrm{-cm}$ line once during these trials. The pseudoconfederates in the D-R group were rewarded by E saying "good" after each judgment involving a $15.5 \mathrm{~cm}$ horizontal line. The Os were called on to respond during the other 15 dissonance trials. Nine of these trials involved a horizontal line that was $23.5 \mathrm{~cm}$ long, five trials used a $19.5-\mathrm{cm}$ line, and the remaining trial used a $15.5-\mathrm{cm}$ line. The order in which the students were called on and the order of presenting the horizontal lines to the pseudoconfederates and Os was random.

\section{RESULTS AND DISCUSSION}

Figure 1 shows ogives fitted to the proportion of times that each of the horizontal lines was judged longer by pseudoconfederates in the D-R and D-NR groups and by their counterparts in the control group during pre- and postdissonance trials. An analysis of variance, with groups as a between-Ss effect and pre- and postdissonance conditions and stimuli (length of horizontal lines) as within-Ss effects, of these data indicated that the Groups by Pre-Post by Stimuli interaction was significant $\quad(F=3.03, \quad d f=20 / 360$, $\mathrm{p}<.01)$. Inspection of the curves shown in Fig. 1 suggests that the significant triple interaction was due to the small differences between pre- and postdissonance conditions in the D-R and D-NR groups and to the pre- to postdissonance reduction in the proportion of horizontal longer judgments in the control group. Additional analyses of variance, Pre- and Postdissonance Conditions by Stimuli by Ss for each group, supported these observations. That is, the pre-post effect and the Pre-Post by Stimuli interaction were not significant in either the D-R or the D-NR groups, but the pre-post effect $(\mathrm{F}=7.12, \mathrm{df}=1 / 12, \mathrm{p}<.05)$ and the Pre-Post by Stimuli interaction $(\mathrm{F}=4.59$, $\mathrm{df}=10 / 120, \mathrm{p}<.01)$ were both significant in the control group.

Inspection and analysis of the judgments made by the counterparts of the pseudoconfederates in the control group indicates the presence of a practice effect. That is, the vertical-horizontal illusion was reduced in the sense that the horizontal lines were less likely to be judged longer than the vertical line during the postdissonance trials than during the predissonance trials.

In contrast, inspection and analysis indicated that the accuracy of the judgments made by pseudoconfederates in the D-R and D-NR groups did not improve during the postdissonance trials. That is, the vertical-horizontal illusion was maintained at about the same level during the pre- and postdissonance trials. This finding suggests that the practice effect may have been counteracted by the pseudoconfederates' attempts to reduce the dissonance aroused by their inconsistent judgments. The pseudoconfederates, for example, could reduce dissonance during the postdissonance trials by distorting the horizontal line so that it would appear to be consonant with their inconsistent judgments. This distortion would be likely to counteract any improvement in the accuracy of postdissonance judgments that would result from additional practice. Thus, the results of the present experiment can be interpreted as supporting one of the derivations from dissonance theory.

The results of the present experiment do not, however, support the Festinger \& Carlsmith (1959) derivation from dissonance theory. This derivation, when applied to the present situation, implies that the pseudoconfederates in the D-R group would use the verbal rewards to justify their inconsistent judgments and, in this way, eliminate or reduce the dissonance aroused by these judgments. If this were the case, the pseudoconfederates 
in the D-R group would be expected to show a practice effect because there would be no need for these students to reduce dissonance by distorting their perception of the horizontal line. As shown above, however, inspection and analysis of the data indicated the absence of a practice effect in the D-R group, and thus, the results of the present experiment fail to support the Festinger \& Carlsmith (1959) derivation.

REFERENCES

FESTINGER, L. $A$ theory of cognitive dissonance. Evanston. III: Row, Peterson, 1957.

IFSTINGIR, L.. \& CARLSMITH, J. M. Cognitive consequences of forced compliance. Journal of Abnormal \& Social Psychology, 1959. 58. 203-211.

HELMREICH, R., \& COLLINS, B. E. Studies in forced compliance: Commitment and magnitude of inducement to comply as determinants of opinion change. Journal of Personality \& Social Psychology, 1968, 10, 75-81.

ROSENBERG, .1. J. When dissonance fails: On eliminating evaluation apprehension from attitude measurement. Journal of Personality \& Social Psychology, 1, 28-43.

\title{
Context effects in impression formation as a function of context source*
}

\author{
WILLIAM GRIFFITT and THOMAS JACKSON \\ Kansas State University, Manhattan, Kans. 66502
}

Context effects in impression formation were studied as a function of the source of contextual stimuli. Assimilation effects were obtained when contextual stimuli and test stimuli were attributed to the same target person. A weak tendency toward contrast effects was noted when contextual stimuli and test stimuli were attributed to separate individuals.

In the investigations of impression formation utilizing the adjective-trait paradigm initiated by Asch (1946), context effects refer to systematic displacements of ratings of test stimuli toward or away from values of context adjectives. Assimilation or "positive context effects" (Anderson \& Lampel, 1965) refer to test stimulus rating displacements toward the context values while contrast or "negative context effects" refer to displacements of test stimulus ratings away from contextual values. Anderson (1962, 1966) has described a weighted averaging model of impression formation that predicts that the rating given to a test stimulus is an averaged function of the context-free value of the stimulus and the impression created by the context stimuli. The model predicts only assimilation or positive context effects and has received support in numerous studies (Anderson \& Lampel, 1965; Kaplan, 1969).

In this paradigm, Ss typically are presented a list of adjectives descriptive of a person and asked to rate the likability of

\footnotetext{
*This research was supported in part by Research Grant MH 16351-02 from the National Institutes of Mental Health. The authors would like to thank Russell Veitch for his assistance.
}

the person as well as the likability of one of the trait adjectives (test stimulus). Thus, both the context and test stimuli are attributed to a single target person and may be considered a within-target-person (WTP) design.

The present study was designed to examine WTP context effects and context effects in a between-target person (BTP) design, in which contextual adjective traits are attributed to one target person while the test stimulus is attributed to a second person. Evidence from studies of interpersonal attraction (Griffitt ${ }^{1}$ ) indicates that, when positive or negative stimulus components attributed to one target person are presented in a context of stimuli attributed to a second target person that is opposite in valence from the first, ratings of each target person are displaced away from ratings obtained when both sets of stimuli are similar in valence. That is, responses to positive targets in a negative-target context were more positive than to positive targets in a positive-target context. Complementary displacements were noted in ratings of negatively described targets in the appropriate contexts. Thus, in BTP designs in which the context stimuli were attributed to separate target persons, contrast effects were obtained, as opposed to the usual assimilation effects noted in WTP designs. Since the Griffitt ${ }^{1}$ study involved ratings of target persons, rather than ratings of single target adjective stimuli, the present investigation was undertaken to compare WTP and BTP context effects in the usual trait-adjective paradigm.

\section{METHOD}

Ss for the experiment were 32 (16 male, 16 female) introductory psychology students at Kansas State University. Context traits of high (H) and low (L) favorability were chosen randomly from an extensive list of prescaled traits (Anderson, 1968). Target stimuli of moderate favorability $(\mathrm{M}+)$ and moderate unfavorability $(\mathrm{M}-$ ) were also chosen from the same list. Single $M+$ or $M-$ target traits were paired randomly with two $\mathrm{H}$ or $\mathrm{L}$ context traits and typed in randomly determined orders on $3 \times 5$ cards. Additionally, single $\mathrm{M}+$ or $\mathrm{M}-$ test adjectives were typed on separate $3 \times 5$ cards. Two replications of each of the four three-trait sets were constructed, each trait being selected without replacement.

One replication of each of the three-trait sets was then paired randomly with one of the four single $M+$ target traits, while the second replication of each three-trait set was paired randomly with one of the four single $\mathrm{M}$ - target traits. Each pair was thus composed of one three-trait set and one single-trait set typed on separate cards and presented to Ss by an overhead projector. Each $S$ was exposed to each pair with the order of presentation of pairs randomized. On each three-trait card a different alphabetic letter was typed, while differing numbers were typed on each single-trait card. Ss were told that each three-trait set represented traits of a given person, each trait ascribed by a different acquaintance, and that the single trait represented a trait ascribed to a different person, also by an acquaintance. Upon presentation of a given trait-set pair, each $\mathrm{S}$ was first asked to rate the favorability of the test trait indicated by $E$ from the three-trait set, then the favorability of the single trait (test) on a 20-point scale. Thus, in the present design, the two context adjectives in the three-trait sets served as WTP contexts for ratings of the target traits of the three-trait sets and as BTP contexts for ratings of the single-target traits attributed to the second target person.

\section{RESULTS AND DISCUSSION}

Mean ratings of the $\mathrm{M}+$ and $\mathrm{M}$ - test traits in each of the two contexts for each of the two context sources are shown in Table 1. Analysis of variance of these data revealed significant effects due to context $(F=5.08, d f=1 / 31, p>.05)$, test trait $(F=26.32, d f=1 / 31, p>.001)$, and the interaction of Context by Source of 